\title{
The contribution to the modal analysis using an infrared camera
}

\author{
Vladimír Dekys ${ }^{1, *}$, Zuzana Stankovicova ${ }^{1}$, Pavol Novak $^{1}$, Ondrej Stalmach ${ }^{1}$ \\ ${ }^{1}$ Faculty of Mechanical Engineering, Department of Applied Mechanics, University of Zilina, \\ Univerzitna 1, 01026, Slovakia
}

\begin{abstract}
The paper deals with modal analysis using an infrared camera. The test objects were excited by the modal exciter with narrowband noise and the response was registered as a frame sequence by the high speed infrared camera FLIR SC7500. The resonant frequencies and the modal shapes were determined from the infrared spectrum recordings. Lock-in technology has also been used. The experimental results were compared with calculated natural frequencies and modal shapes.
\end{abstract}

Keywords: infrared camera, vibration, modal analysis, resonance, modal shape

\section{Introduction}

When an object is excited, for example by acting on forces in the deformation object, part of the energy supplied is also radiated in the infrared frequency range by the detectable infrared camera. This phenomenon is used, for example, in detecting Luders bands [1], nondestructive material testing $[2,3]$ or detection of plastic areas around stress concentrators. The temperature of the object is then determined after the use of calibration relationships, the relationship between the level of infrared radiation build up and the black body temperature. If this excitation causes only elastic deformations in the test, and if the load frequency is high enough to meet adiabatic conditions, then the relationship between temperature change and the change of trace of the stress tensor $\Delta(\operatorname{tr} \sigma)$ can be expressed according to the relationship $[4,5]$ :

$$
\Delta T=-\frac{\alpha}{\rho C_{p}} T_{0} \Delta(\operatorname{tr} \boldsymbol{\sigma})
$$

$\Delta(\operatorname{tr} \sigma)$ is the response of the object to the stimulus forces, $\alpha$ is the linear coefficient of thermal expansion; $C_{p}$ is the heat capacity at the constant pressure, $\rho$ is density of the analysed object, $T_{0}$ is starting temperature. Adiabatic conditions mean a sufficiently high object load frequency for steel greater than $2 \mathrm{~Hz}$, for aluminium alloys greater than $20 \mathrm{~Hz}$.

Changing the temperature is often very small, for a steel change of the stress by $1 \mathrm{MPa}$ will cause a change of about $1 \mathrm{mK},[6]$. Such a change is, however, at the infrared camera noise level, and its detection may require a specific procedure to increase the signal/noise

\footnotetext{
* Corresponding author: vladimir.dekys $@$,fstroj.uniza.sk

Reviewers: Ivan Kuric, Grzegorz Domek
} 
ratio, e.g. using lock-in method $[3,7]$ to determination of change of temperature. In the case of determination of temperatures it is important to know the emissivity of the tested objects $[8]$.

\section{Experiment}

If, for example, an impulse hammer is used as a stimulus, then we are able to excite the object within a certain frequency range, depending on the used hammer tip, respectively from the duration of the generated pulse. This can also be achieved by excitement of white noise in the same frequency range, although finding the acquired dependencies may require a greater number of averages. Upon exciting the above mentioned noise, the object vibrates at resonant frequencies, corresponding to its natural frequencies. Excited shapes associated with individual frequencies determine the resulting vibration of the object. Subsequently, the vibration of an object causes changes in its deformations, and there is also a change in the radiated energy detected by the infrared camera.

The frequency response function FRF is generally defined, for example, as the ratio of the output response spectrum of the system to the input stimulus spectrum. In our case, the output signal is measured by the IR camera and the input spectrum was considered to be constant in the frequency range of exciting white noise. Experimental determination of resonant frequencies is based, for example, on the determination of peaks at frequencies in the amplitude spectrum. The corresponding shapes to their natural frequencies (resonance frequencies) are determined by the imaginary spectrum of the frequency response function. The imaginary spectrum was preferred when processing the camera outputs because this spectrum corresponds to the deformations and thus to the changes in the stress field and consequently to the changes in infrared spectral radiations that are registered by the camera.

Two experiments were performed. In the first case, a rectangular S235J steel sheet with dimensions of 160x60 mm with a thickness of 1mm was used. The plate was excited by the TIRA200 modal exciter in Figure 1. The plate was screwed directly onto the moving core of the modal exciter. The input signal was narrowband white noise in the range of 10 $500 \mathrm{~Hz}$ in Figure 2. The radiation of vibration sheet was registered in mid wavelength (MWIR) using the FLIR SC7500 at native frame rate of $383 \mathrm{~Hz}$. The record was made in ResearchIR Max and then imported and processed in MATLAB.

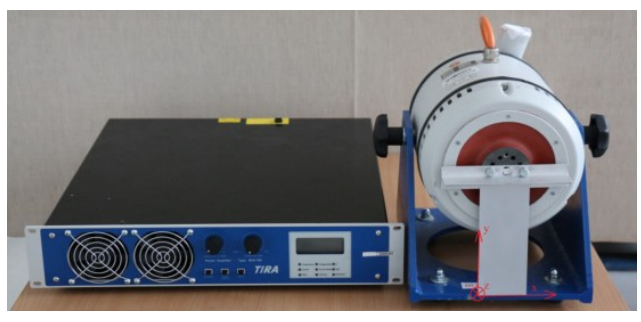

Fig. 1. The test specimen excited by TIRA200, cause 1 , the plate was screwed directly onto the moving core of the modal exciter

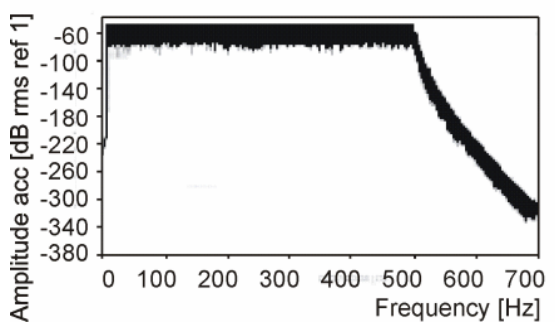

Fig. 2. The spectrum of input signal was narrowband white noise in the spectral range of $10-500 \mathrm{~Hz}$

The sequence of frames was processed by the lock-in method. This method is a specific form of Fourier Transform. In the case of the classical transformation the minimum frequency of the Fourier series is determined based on the number of samples and sampling frequency. For the lock-in method this frequency is equal to the load frequency. Subsequently, if the response to the input frequency $f_{0}$ of the narrowband white noise is to 
be determined then this frequency is the lowest frequency of the Fourier series for lock-in, $f_{0}=f_{\text {lockin }}$.

It is assumed that the system is stimulated by the load force with the frequency $f_{0}$ and the response of the system $s(t)$ sufficiently describes the harmonic function with frequency $f_{\text {lockin }}=f_{0}$, amplitude $A$ and phase shift $\varphi$

$$
\begin{gathered}
s(t)=A \sin \left(2 \pi f_{\text {lockin }} t+\varphi\right)=a \cos \left(2 \pi f_{\text {lockin }} t\right)+b \sin \left(2 \pi f_{\text {lockin }} t\right), \\
a=A \sin (\varphi), b=A \cos (\varphi) .
\end{gathered}
$$

Let the amplitude of this response $s(t)$ is very small and the signal $S(t)$ being measured also contains a much higher noise $N(t)$, according to the relationship

$$
S(t)=s(t)+N(t)
$$

If $S(t)$ is expressed by Fourier's series than

$$
\begin{aligned}
S(\mathrm{t}) & =\frac{A_{0}}{2}+\sum_{k=1}^{+\infty} A_{k} \sin \left(2 \pi k f_{\text {lockin }} t+\varphi_{k}\right)= \\
& =A_{1} \sin \left(2 \pi f_{\text {lockin }} t+\varphi_{1}\right)+\left[\frac{A_{0}}{2}+\sum_{k=2}^{+\infty} A_{k} \sin \left(2 \pi k f_{\text {lockin }} t+\varphi_{k}\right)\right]= \\
& =s(t)+N(t) .
\end{aligned}
$$

If $A=A_{l}$ and $j=j_{l}$, than the coefficients $a, b$ in (2) can be estimated by using the relationships of Discrete Fourier transformation

$$
a=\sum_{k=0}^{N-1} S_{i} \cos \left(2 \pi k f_{\text {lockin }} \Delta t\right), \quad b=B_{k} \sin \left(2 \pi k f_{\text {lockin }} \Delta t\right)
$$

where $S_{k}$ is the $k$-th frame recorded by infrared camera (the frames are numbered $0, \ldots N-1), \Delta t$ is the period of frame frequency of camera. By using $a, b$, the amplitude $A$, phase $\varphi$, the real and the imaginary part of $s$ were determined for $f_{\text {lockin }}$.

The region of interest (ROI) was selected in the image. For the selected $f_{\text {lockin }}$, an imaginary part of $s$ was determined for this ROI by the lock-in method. Subsequently, the variance of the values obtained in this ROI was determined, that is, a pair of values (variance, $f_{\text {lockin }}$ ). The procedure was repeated for different frequencies $f_{\text {lockin }}$ and the resulting data set is shown in Figure 3.

In Figure 4 is the narrowband response spectrum obtained by processing the vibration velocity measurement by Laser Doppler Vibrometer LDV100.The numerical values of the resonant frequencies determined by IR camera and vibrometer measurements as well as the results of the natural frequencies determined by the ANSYS modal analysis for the given object are presented in Table 1.

A comparison of the resonant and the natural frequencies has been demonstrated for frequencies No. 1, 3 and 5. Frequencies No. 2 and 4 were not detected by an infrared camera because a small value of $\Delta(\operatorname{tr} \sigma)$ was due to the presence of torsion. Frequencies No. 2 and 4 were not detected by the vibrometer because this device is primarily sensitive to the vibration in a plane perpendicular to the direction of the laser beam and may therefore be less sensitive to the torsion of the sample.

Determination of extreme variance values for the imaginary part versus different $f_{\text {lockin }}$ was used to determine resonance frequencies. The extreme variance value is given by the excitation of the corresponding oscillation shape, which is reflected in the response of the object. By this procedure, it was not possible to detect other than bending modal shapes, 
because torsion modal shapes did not cause a sufficient change in the principal stresses (Fig. $5-18$ ).

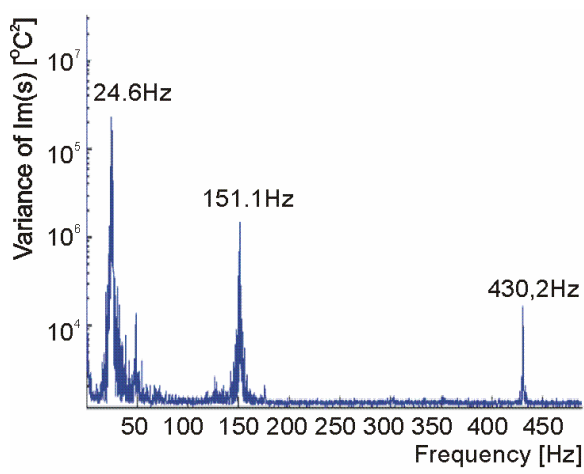

Fig. 3. The spectrum of variance from IR camera

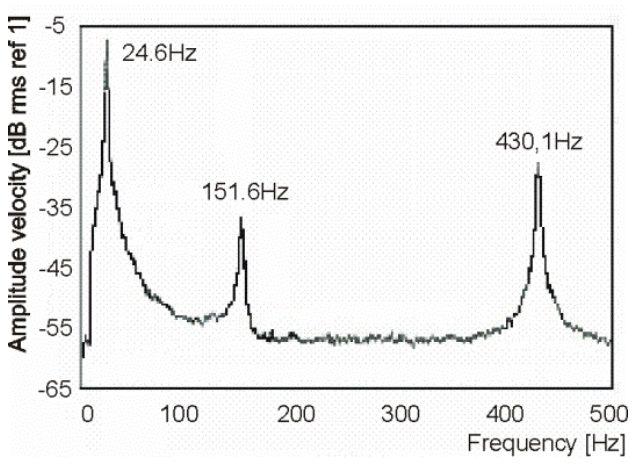

Fig. 4. The spectrum of velocity of vibration from laser beam vibrometer

Table 1. The comparison of resonant frequencies determined by IR camera, laser beam vibrometer and FEA ANSYS

\begin{tabular}{|l|c|c|c|c|c|}
\hline & \multicolumn{5}{|c|}{ Frequency [Hz] } \\
\hline Frequency No. & 1. & 2. & 3. & 4. & 5. \\
\hline Infrared camera & 24.6 & - & 151.1 & - & 430.2 \\
\hline LD vibrometer & 24.6 & - & 151.5 & - & 430.1 \\
\hline Modal analysis, FEA & 24.3 & 125.2 & 152.2 & 399.9 & 429.1 \\
\hline
\end{tabular}

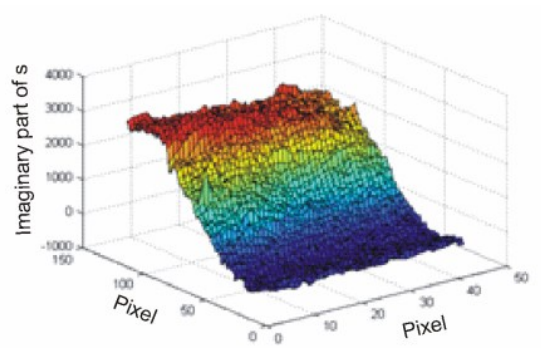

Fig. 5. Deformed bending shape at resonant frequency $24.6 \mathrm{~Hz}$, IR camera, No. 1, axonometric view

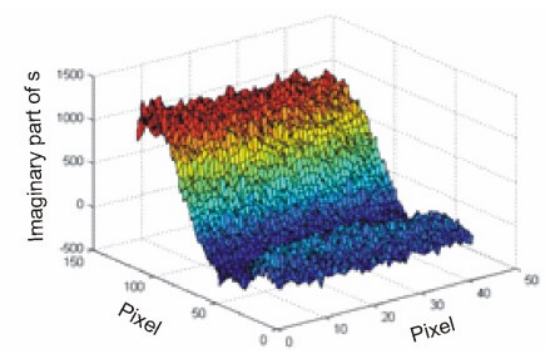

Fig. 7. Deformed bending shape at resonant frequency $151.1 \mathrm{~Hz}$, IR camera, No. 3, axonometric view

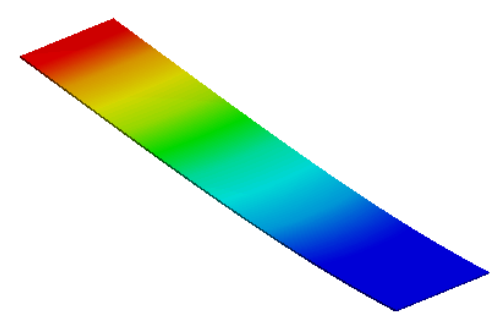

Fig. 6. Modal bending shape at natural frequency $24.3 \mathrm{~Hz}$, FEA ANSYS, No. 1, axonometric view

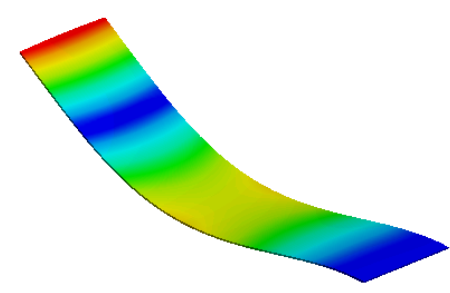

Fig. 8. Modal bending shape at natural frequency 152.2 Hz, FEA ANSYS, No. 3, axonometric view 


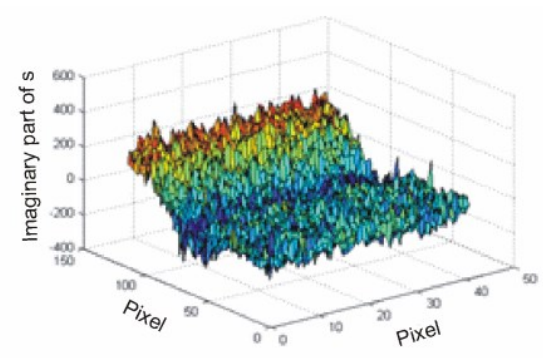

Fig. 9. Deformed bending shape at resonant frequency $430.2 \mathrm{~Hz}$, IR camera, No. 5 , axonometric view

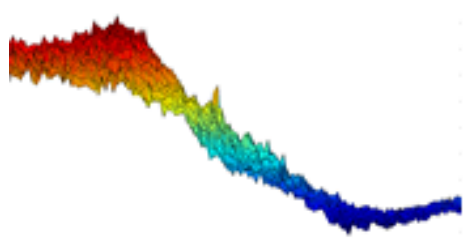

Fig. 11. Deformed bending shape at resonant frequency $24.6 \mathrm{~Hz}$, IR camera, No. 1, a side view

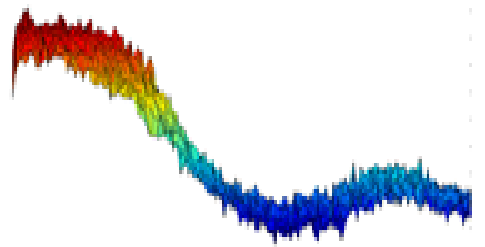

Fig. 13. Deformed bending shape at resonant frequency $151.1 \mathrm{~Hz}$, IR camera, No. 3, a side view

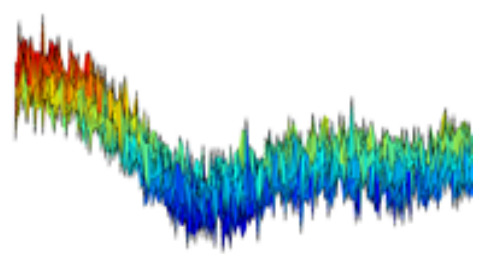

Fig. 15. Deformed bending shape at resonant frequency $430.2 \mathrm{~Hz}$, IR camera, No. 5, a side view

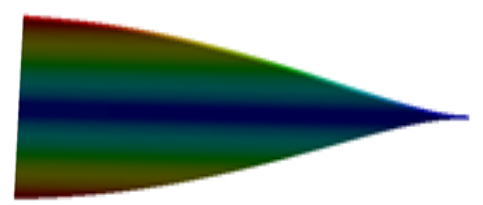

Fig. 17. Modal torsional shape at natural frequency $125.2 \mathrm{~Hz}$, FEA ANSYS, No. 2, a side view, the shape not detected by the IR camera

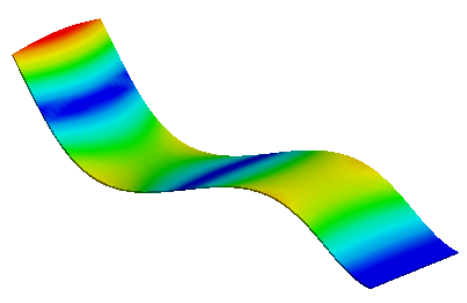

Fig. 10. Modal bending shape at natural frequency $429.1 \mathrm{~Hz}$, FEA ANSYS, No. 5, axonometric view

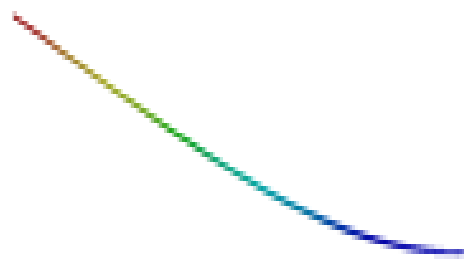

Fig. 12. Modal bending shape at natural frequency $24.3 \mathrm{~Hz}$, FEA ANSYS, No. 1, a side view

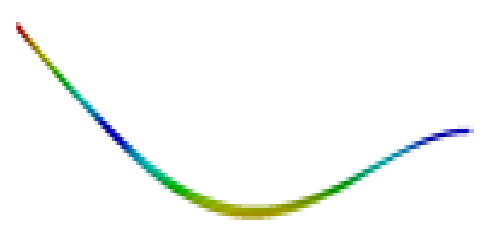

Fig. 14. Modal bending shape at natural frequency $152.2 \mathrm{~Hz}$, FEA ANSYS, No. 3, a side view

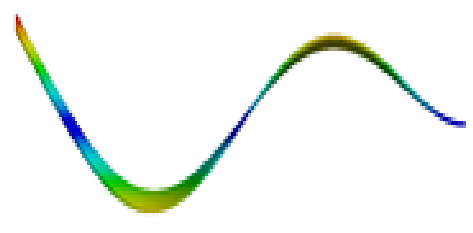

Fig. 16. Modal bending shape at natural frequency $429.1 \mathrm{~Hz}$, FEA ANSYS, No. 5, a side view

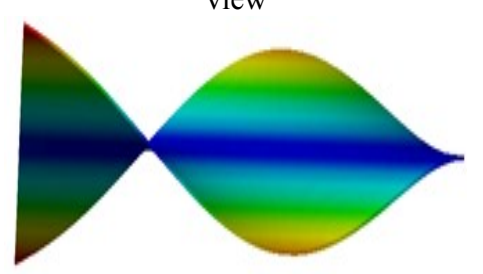

Fig. 18. Modal torsional shape at natural frequency $399.9 \mathrm{~Hz}$, FEA ANSYS, No. 4, a side view, the shape not detected by the IR camera 
Experiment No. 2 measured the vibration of the plastic propeller of the table fan. The bandwidth excitation spectrum is again narrowband in the range of 10-192 Hz, measured by an infrared camera and a laser vibrometer. ANSYS was used to calculate the natural frequency of the fan. Larger discrepancies were recorded between the specified first resonant frequency: the IR camera $(43.8 \mathrm{~Hz})$, the laser vibrometer $(42.1 \mathrm{~Hz}-42.9 \mathrm{~Hz})$, and the frequency specified in the FEA ANSYS $(45.7 \mathrm{~Hz})$. In Figure 19 and 20 the modal shape for these frequencies are shown. Although the IR camera output is not as brilliant as the FEA, it is possible to observe the bend of propeller at this frequency as determined by the experiment and the FEA. Frequency deviations and a less clear reconstruction of the oscillation shape attribute the blade curvature and the need for a better identification of the FEA material model.

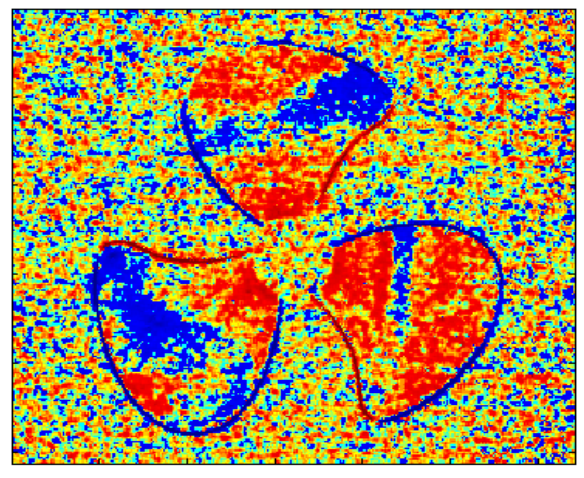

Fig. 17. Deformed bending shape at resonant frequency $43.8 \mathrm{~Hz}$, IR camera, a front view

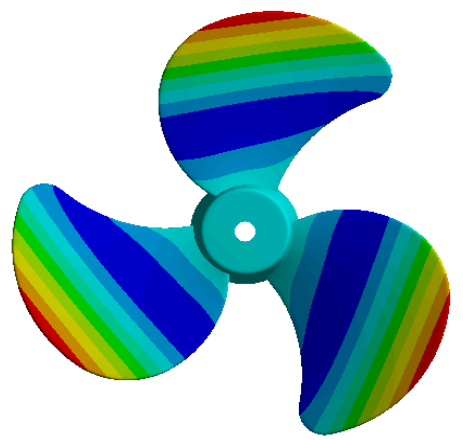

Fig. 18. Modal bending shape at natural frequency $44.7 \mathrm{~Hz}$, FEA ANSYS, a front view

\section{Conclusion}

In the paper was presented an approach to determine resonant frequencies and corresponding natural frequencies by means of radiation detection in the mid infrared band (MWIR). Resonant frequencies were determined using lock-in technology. The variance of imaginary parts for individual pixels in the region is interesting. The peak values of this variance were used for resonant frequency detection. We consider the match between IR camera, laser vibrometer and ANSYS is sufficient.

Corresponding modal shapes were obtained for these frequencies. In the measurements, the objects were excited by narrow-band white noise.

In this contribution, smoothed or approximate modal shapes were not used, as we would like to analyse the properties of excitation signal on the quality of the response in future.

This contribution is related to dynamic analysis work [9, 10] at the Faculty of Mechanical Engineering, Department of Applied Mechanics, University of Zilina and [11, 14].

This work was supported by the Slovak Research and Development Agency under the contract No. APVV-0736-12 and by VEGA 1/0795/16, KEGA 017ŽU-4/2017.

\section{References}

1. V. Dekys, P. Kopas, M. Sapieta, O. Stevka, A detection of deformation mechanisms using infrared thermography and acoustic emission. Applied Mechanics and Materials 474, 315-320 (2014) 
2. Z. Stankovicova, V. Dekys, F. Novy, P. Novak, Nondestructive testing of metal parts by using infrared camera. Procedia Engineering 177, 562-567 (2017)

3. A. Mendioroz, A. Castelo, R. Celorrio, A. Salazar, Defect Characterization from Lockin Vibrothermography Data. Int J Thermophys 36, 1208-1216 (2015)

4. X. P. V. Maldague, Theory and Practice of Infrared Technology for Nondestructive Testing. (Wiley, New York 2001)

5. M. Sapieta, V. Dekys, A. Sapietova, Thermal-stress analysis of beam loaded by 3 point bending. Procedia Engineering 136, 216-219 (2016)

6. P. Bremond, New developments in ThermoElastic Stress Analysis by Infrared Thermography. (IV conferencia Panamericana de END. Buenos Aires, 2007)

7. O. Breitenstein, Lock-in thermography. (Springer, New York,: Springer. 2010)

8. M. Honner, P. Honnerova, Survey of emissivity measurement by radiometric methods. Applied Optics 54, 669-683 (2015)

9. M. Zmindak, Z. Pelagic, Modeling of shock wave resistance in composite solids Procedia Engineering 96, 517-526 (2014)

10. A. Sapietova, R. Petrech, M. Petrovic, Analysis of the dynamical effects on housing of the axial piston hydromotor. Applied Mechanics and Materials 474, 357-362 (2014)

11. J. Kortis, L. Daniel, M. Skarupa, Experimental modal test of the laboratory model of steel truss structure. Civil and Enviromental Engineering 12 (2), 116-121 (2016)

12. J. Bocko, V. Nohajova, T. Harcarik, Symmetries of differential equations describing beams and plates on elastic foundations. Procedia Engineering 48, 40-45 (2012)

13. F. Klimenda, J. Soukup, Modal analysis of thin aluminium plate. Procedia Engineering 177, 11-16 (2017)

14. M. Nad, L. Rolnik, L. Cimancova, Conference: Prediction of Changes in Modal Properties of the Euler-Bernoulli Beam Structures Due to the Modification of Its Spatial Properties. International Journal of spatial Properties 17 (1740014), (2017) 